\title{
Numerical Investigation of Separated Solitary Waves Solution for KDV Equation through Finite Element Technique
}

\author{
S. Kapoor \\ Department of Mathematics, \\ THDC Institute of Hydropower \\ Engineering and Technology, \\ B.Puram, Tehri
}

\author{
S. Rawat \\ Department of Mathematics, \\ Galgotias University, G. Noida
}

\author{
S. Dhawan \\ Department of Mathematics \\ National Institute of Technology \\ Jalandhar,
}

\begin{abstract}
The Present manuscript reports the solution of well known non linear wave mechanics problem called KDV equation, here main emphasis is given on the Mathematical modeling of traveling waves and their solutions in the form of Kortewegde Vries equation (KdV) It is a non-linear Partial Differential Equation (PDE) of third order which arises in a number of physical applications such as water waves, elastic rods, plasma physics etc. We present numerical solution of the above equation using B-spline FEM (Finite Element Method) approach. The ultimate goal of the paper is to solve the above problem using numerical simulation in which the accuracy of computed solutions is examined by making comparison with analytical solutions, which are found to be in good agreement with each other along with that we discussed the physical interpolation of the soliton study in which we found that the travel waves reaches to the maximum magnitude of the velocity in the short time of the interval and there is an uncertainty in the motion of the moving waves. Another important observation we found that the maximum magnitude of the velocity in the most of the time domain is around 1 but in some of the condition waves having a unnatural phenomena which is called the existence of the doubly soliton is seemed frequently. All above observation which is clearly indication of the generic outcome of a weakly nonlinear long-wave asymptotic analysis of many physical systems. The another achievement of the work is to implementation of the cubic Bspline FEM in the above non linear propagating waves phenomena.
\end{abstract}

\section{Keyword}

B-Spline, FEM, KDV, Separated solitary Waves

\section{INTRODUCTION}

In engineering and real world science the wave is a disturbance that travels through space and time and the different kind of waves is occur in nature every part of flow dynamics having a different kind of application in nature. The term wave is often intuitively understood as referring to a transport of spatial disturbances that are generally not accompanied by a motion of the medium occupying this space as a whole. The modeling of the each one is in different form. Here our objective is to introduce one of the kind of wave mechanics problem called KDV equation. In mathematics, the Korteweg-de Vries equation (KdV equation for short) is a mathematical model of waves on shallow water surfaces. It is particularly notable as the prototypical example of an exactly solvable model, that is, a non-linear PDE whose solutions can be exactly and precisely specified. The solutions in turn include prototypical examples of solitons. KdV can be solved by means of the inverse scattering transform. The mathematical theory behind the $\mathrm{KdV}$ equation is rich and interesting, and, in the broad sense, is a topic of active mathematical research. The equation is named for Diedrik Korteweg and Gustavde Veris who studied it in (Kortewegde Vries 1895) [1], though the equation first appears in Boussineq [2], Zabusky, N. J.; Kruskal, M. D. [3] observed unusual nonlinear interaction among "solitary-wave puses" propagating in nonlinear dispersive media, Miura et.al [4] gives Korteweg-de Vries equation and generalizations. II. Existence of conservation laws and constants of motion along with Lax [5] find the integral solution of non linear solitary wave equation then Vliegenthart [6] find the solution of KDV equation using FDM approach, hence Miles., John W. [7] gives a detail of KDV equation as an essay then Dingmans, M.W. [8] founds a Water wave propagation over uneven bottoms using numerical simulation, de Jager, E.M. [9]. Reports the detail origin of the KDV equation it imp aspects and there historical background and then Darvishi [10] gives a Numerical Solution of the Lax's 7th-order KdV Equation by Pseudo spectral Method now I $\mathrm{n}$ this paper we have choose $\mathrm{KdV}$ equation as our model problem as it is used in many different fields to model various physical phenomena of interest. In 1895 Korteweg [1] showed that long waves, in water of relatively shallow depth, could be described approximately by a nonlinear equation of the form

$$
u_{t}+\left(c_{0}+c_{1} u\right) u_{x}+v u_{x x x}=0
$$

where $c_{0}, c_{1}, v$ are real constants. and can be used as a model describing the lossless propagation of shallow water waves. It is well known that the Korteweg-de Vries equation is the generic outcome of a weakly nonlinear long-wave asymptotic analysis of many physical systems. It is categorized by its family of solitary wave solutions, with the familiar $\operatorname{sech}^{2}$ profile. It has also been used as a model for ion-acoustic waves in plasma, pressure waves in liquid-gas bubble mixtures rotating flow down a tube and thermally excited phonon packets in low-temperature nonlinear crystals [10 11]. The aim of present paper is to focus on the numerical solution of KDV equation using finite element technique with B-spline functions, splines play an important role in computational study and visulization [13]. 
The Korteweg-de Vries (KDV) equation

$$
u_{t}+u_{x}+\alpha u u_{x}+\beta u_{x x x}=0 \text {, }
$$

where $\alpha \beta$ are real constants, was first introduced as a model describing the lossless propagation of shallow water waves [1]. Since then it has been used as a model for ion-acoustic waves in plasma ,[10-12] pressure waves in liquid-gas bubble mixtures [14] rotating flow down a tube and [15] thermally excited phonon packets in low-temperature nonlinear crystals. It is well known that the Korteweg-de Vries equation is the generic outcome of a weakly nonlinear long-wave asymptotic analysis of many physical systems. It is categorized by its family of solitary wave solutions, with the familiar sech $^{2}$ profile.

Due to its properties, the KdV equation was the source of many applications and results in a large area of nonlinear physics. For example the $\mathrm{KdV}$ equation and its generalizations. Also another formulation of (1) is as

$$
u_{t}+\alpha u u_{x}+\beta u_{x x x}=0, a \leq x \leq b
$$

with boundary conditions

$$
u(a, t)=u(b, t)=0, u_{x}(a, t)=u_{x}(b, t)=0
$$

Zabusky and Kruskal [15] solved the KdV equation using a finite difference explicit method with periodic boundary conditions and showed the existence of solitons which propagated with their own velocities, exerting essentially no influence on each other. The Fourier expansion procedure [16] which is competitive with finite difference method is used to study numerically the $\mathrm{KdV}$ equation. Taha and Ablowitz [17- 18] have done excellent comparisons between different known schemes and their scheme for $\mathrm{KdV}$ equation which is developed using notions of the inverse scattering transform. An effiient numerical method is developed for solving the KdV equation by Fornberg and Whitham [19]. Iskander [20] studied the KdV equation numerically using an implicit finite difference scheme based on the combined approach of linearization and finite difference method. Karakashian and McKinney [21] obtained optimal rate of convergence estimates in time for high-order fully discrete approximations to the $\mathrm{KdV}$ equation with periodic boundary conditions. These approximations are generated by a finiteelement process [22] for the spatial discretization and implicit Runge-Kuttamethod [23] for the time stepping. After that S.Kapoor [24] has an attempted to solve well known burgers equation using B-spline FEM technique this is also a good achievement due the remarkable accuracy and agreement with the exact solution is found in the further stage V.Dabral [2426] is introduce the b-spline FEM for the solution of wave mechanics problem like MEW, and KDV equation, here the objective is very clear that was to implementation of the method in those type of problem with special case and they successfully implemented spline function as basis function with homogenous boundary condition, taken care of the above we keep in mind the work of Gupta and Kumar [27] in which employs cubic trigonometric B-spline to solve linear two point second order singular boundary value problems for ordinary differential equations. The objective of the present work is not only to introduce a finite element technique for the numerical solution of KDV equation using B-spline functions but also to understand the behavior of the these type of wave problem which in general come into the nature such as shock wave , true waves found in dispersive medium it highly non linear also, so here the attempt is to find out the solution of non linear PDE using B-spline FEM is taken.

\section{FINITE ELEMENT SOLUTION}

A general form of KDV equation is taken for the present study

$$
\frac{\partial u}{\partial t}+\varepsilon u \frac{\partial u}{\partial x}+\mu \frac{\partial^{2} u}{\partial x^{2}}=0
$$

with the boundary conditions

$$
\begin{aligned}
u(a, t)=u(b, t) & =0 \\
\frac{\partial u}{\partial x}(a, t)=\frac{\partial u}{\partial t}(b, t) & =0
\end{aligned}
$$

where $\varepsilon, \mu$ are positive parameters. Let us consider $x_{0}<x_{1}<$ $\cdots<x_{N}$ be the partition of [a; b] by the knots $x_{i}$. Cubic Bsplines are used to approximate the solution $\mathrm{u}(\mathrm{x}, \mathrm{t})$. Thus the set of splines $B_{-1}, B_{0}, \ldots B_{N}, B_{N+1}$ forms a basis for functions defined over $[\mathrm{a}, \mathrm{b}]$. Cubic B-splines $B_{m} ;(m=-1, \ldots N+$ 1)at knots $x_{m}$ to form a basis over the problem domain are defined by [4]

$$
B_{m}(x)=\frac{1}{h^{3}}\left\{\begin{array}{rc}
f_{1}(x) & x \in\left[x_{m-2}, x_{m-1}\right] \\
f_{2}(x) & x \in\left[x_{m-1}, x_{m}\right] \\
f_{3}(x) & x \in\left[x_{m}, x_{m+1}\right] \\
f_{4}(x) & x \in\left[x_{m+1}, x_{m+2}\right] \\
0 & \text { otherwise }
\end{array}\right.
$$

Where

$$
\begin{aligned}
& f_{1}(x)=\left(x-x_{m-2}\right)^{3} \\
& f_{2}(x)=h^{3}+3 h^{2}\left(x-x_{m-1}\right)+3 h\left(x-x_{m-1}\right)^{2}- \\
& 3\left(x-x_{m-1}\right)^{3} \\
& f_{3}(x)=h^{3}+3 h^{2}\left(x_{m+1}-x\right)+3 h\left(x_{m+1}-x\right)^{2}- \\
& 3\left(x_{m+1}-x\right)^{3} \\
& f_{4}(x)=\left(x_{m+2}-x\right)^{3}
\end{aligned}
$$

where $\mathrm{m}=-1, \ldots, \mathrm{N}+1$ and $h=\left(x_{m+2}-x_{m}\right)$ for all $\mathrm{m}$. We transform the cubic B-splines into element shape functions over the finite intervals $[0, \mathrm{~h}]$ using a local coordinate system $\pi=x-x_{m}, 0 \leq \pi \leq h$ Over $[0, \mathrm{~h}]$ the cubic B-splines in terms of $\pi$ are given by

$$
\begin{aligned}
& B_{m-1}=\left(1-\frac{\pi}{h}\right)^{3} \\
& B_{m}=4-3 \frac{\pi}{h}+3\left(1-\frac{\pi^{2}}{h}\right)^{2}-3\left(1-\frac{\pi}{h}\right)^{3} \\
& B_{m+1}=1+3 \frac{\pi}{h}+3\left(\frac{\pi}{h}\right)^{2}-3\left(\frac{\pi}{h}\right)^{3} \\
& B_{m+2}=\left(\frac{\pi}{h}\right)^{3}
\end{aligned}
$$


Since a finite element $\left[x_{m}, x_{m+1}\right]$ is covered by four successive cubic B-splines, local approximation over each element is given by applying Galerkin method to (2) over each element

$\int_{x_{m}}^{x_{m+1}}\left(\phi\left(\frac{\partial u}{\partial t}+\varepsilon u \frac{\partial u}{\partial x}\right)-\mu \phi_{x} \frac{\partial^{2} u}{\partial x^{2}}\right) d x=0$

We seek the approximation $u_{h}$ to the solution in terms of cubic B-spline basis functions and element parameter $\sigma$ in the form of $u_{h}=\sum_{i=-1}^{N+1} B_{i} \sigma_{i}$ where $B_{m-1} \ldots B_{m+2}$ are B-splines acting as shape functions for each element and $\sigma_{m-1}, \sigma_{m}, \boldsymbol{\sigma}_{\boldsymbol{m + 1}}, \sigma_{m+2}$, are nodal parameters. Using our approximation $u_{h}$ in (7)

$\sum_{j=m-1}^{m+2}\left(\int_{0}^{h} B_{i} B_{j} d x\right) \dot{\sigma}_{j}^{e}+$

$\varepsilon j=m-1 m+2 k=m-1 m+20 h B i B j B k^{\prime} d x \sigma j e \sigma$

$k e-\mu j=m-1 m+20 h B i^{\prime} B j^{\prime \prime} d x \sigma j e$

(8)

The matrix formulation can be represented as

$$
X^{e} \sigma^{e}+\varepsilon Y^{e} \sigma^{e}-\mu Z^{e} \sigma^{e}
$$

Where $X^{e}=\int_{0}^{1} B_{i} B_{j} d x, \quad Y^{e}=\int_{0}^{1} B_{i} B_{j} B_{k}^{\prime} d x, Z^{e}=$ $\int_{0}^{1} B_{i}^{\prime} B_{j}^{\prime \prime} d x$ with i,j,k taking values $\mathrm{m}-1, \mathrm{~m}, \mathrm{~m}+1, \mathrm{~m}+2$ for each element $\left[x_{m}, x_{m+1}\right]$ and the general row of each element matrix is given by

$$
\begin{aligned}
& X=\frac{h}{140}(1,120,1191,2416,1191,120,1) \\
& Y=\frac{h}{40}(-6,-336,-1470,0,1470,336,6) \\
& Z=\frac{1}{2 h^{2}}(-3,-24,57,0,-57,24,3)
\end{aligned}
$$

Assembling contributions from all the elements gives a global system of equations as $X \dot{\sigma}+Y \varepsilon \sigma-\mu \mathrm{Z} \sigma=0$ Using a Crank-Nicolson approach in time the vector $\sigma$ and its time derivative $\dot{\sigma}$ are given by $\sigma=\frac{\sigma^{n}+\sigma^{n+1}}{2}, \dot{\sigma}=\frac{\sigma^{n+1}-\sigma^{n}}{\Delta t}=$ where $\mathrm{n}$ denotes the time level. So, finally we get (9) in the form as

$$
\left[X+\frac{\sigma \Delta t}{2} Y-\frac{1}{2}(\mu \Delta t) Z\right] \sigma^{n+1}=\left[X-\frac{\varepsilon \Delta t}{2} Y-\frac{1}{2}(\mu \Delta t) Z\right] \sigma^{n}
$$

giving recurrence relation for computing $\sigma^{n}$ for different time levels. Initially we calculate $\sigma^{0}$ and with the help of (10) we calculate the first iteration $\sigma_{1}^{1}$ using $\sigma=\sigma^{0}$ Next approximation is calculated using $\frac{1}{2}\left(\sigma^{0}+\sigma_{2}^{1}\right)$. In the similar way we carry on the iteration process and the approximate solution $u^{h}$ is calculated from $\sigma^{n}$.

\section{TEST PROBLEM AND NUMERICAL RESULTS}

In this section, to check the proposed technique we analyses the solution for single soliton as well interaction of two. Solution profiles have been recorded by taking into account different parameters. For the single soliton study, we have the analytical solutions

$$
u(x, t)=A \operatorname{sech}^{2}\left(\kappa x-\omega t-x_{0}\right)
$$

With $A=12 \kappa^{2}, \omega=4 \kappa^{3}$. we take $\kappa=0.3$ and initial condition $x_{0}=0$. Fig. shows the solution for single soliton study with $x_{0}$, corresponding to the exact solution (11) taking $\kappa=0.3,0.2$. Consider KDV equation with $\varepsilon=0.0013010833$ with the initial value of one soliton solution

$$
u(x, t)=3 \operatorname{csech}^{2}\left(\sqrt{\frac{c}{4 \varepsilon}}(x-c t)\right)
$$

where $c=1 / 3$ solution has the advection speed $1 / 3$ and solution region is taken to be $(-1,2)$. Corresponding to the problem with exact solution given by (12), results are shown in taking $\mathrm{c}=1 / 3, \varepsilon=0.0013020833$ at different times. If we consider the third order generalized KDV equation of the form

$$
\begin{aligned}
& u_{t}+u^{p} u_{x}+u_{x x x}=0, \\
& u(x, 0)=\left[\operatorname{Asech}^{2}\left(\kappa x-x_{0}\right)\right]^{1 / p}
\end{aligned}
$$

and available exact solution is of the form

$$
u(x, t)=\left[\operatorname{Asech}^{2}\left(\kappa x-c t-x_{0}\right)\right]^{1 / p}
$$

where $\quad p \geq 2, \kappa, m$ and $x_{0} \quad$ are constants with $A=$ $\frac{2(p+1)(p+2)}{m^{2}} \kappa^{2}, c=\frac{4 \kappa^{2}}{m^{2}}$. In the next part we have the solutions for the interaction of two solitary waves. Next we see interaction of two solitons. To conduct the numerical simulation, we consider some examples for this case. For first example in this study we have the exact solution given by

$$
u(x, t)=12 \frac{x_{1}}{\left(1+e^{\theta_{1}}+e^{\theta_{2}}+a^{2} e^{\theta_{1}+\theta_{2}}\right)^{2}}
$$

Where $X_{1}=\kappa_{1}^{2} e^{\theta_{1}}+\kappa_{2}^{2} e^{\theta_{2}}+2\left(\kappa_{2}-\kappa_{1}\right)^{2} \kappa_{1}^{2} e^{\theta_{1}+\theta_{2}}+$ $a^{2}\left(\kappa_{2}^{2} e^{\theta_{1}}+\kappa_{1}^{2} e^{\theta_{2}}\right) e^{\theta_{1}+\theta_{2}}$

With $\kappa_{1}=0.4, \kappa_{2}=0.6, a^{2}=\left(\frac{\kappa_{1}-\kappa_{2}}{\kappa_{1}+\kappa_{2}}\right)^{2}=\frac{1}{25}, \theta_{1}=\kappa_{1} x-$ $\kappa_{1}^{3} t+x_{1}, \theta_{2}=\kappa_{2} x-\kappa_{2}^{3} t+x_{2}, x_{1}=4, x_{2}=15$ Consider KDV equation with $\varepsilon=0.0013010833$, with the initial value of two solitons, we have

$$
u(x, t)=12 \frac{Y_{1}}{\left(1+e^{\theta_{1}}+e^{\theta_{2}}+a^{2} e^{\theta_{1}+\theta_{2}}\right)^{2}}
$$

Where $Y_{1}=\kappa_{1}^{2} e^{\theta_{1}}+\kappa_{2}^{2} e^{\theta_{2}}+2\left(\kappa_{2}-\kappa_{1}\right)^{2} \kappa_{1}^{2} e^{\theta_{1}+\theta_{2}}+$ $a^{2}\left(\kappa_{2}^{2} e^{\theta_{1}}+\kappa_{1}^{2} e^{\theta_{2}}\right) e^{\theta_{1}+\theta_{2}}$

With $\kappa_{1}=1, \kappa_{2}=1.5, a^{2}=\left(\frac{\kappa_{1}-\kappa_{2}}{\kappa_{1}+\kappa_{2}}\right)^{2}=\frac{1}{25}, \theta_{1}=\kappa_{1} \frac{x}{\sqrt{6 \varepsilon}}-$ $\kappa_{1}^{3} \frac{t}{6^{3 / 2} \sqrt{\varepsilon}}-3, \theta_{2}=\kappa_{1} \frac{x}{\sqrt{6 \varepsilon}}-\kappa_{2}^{3} \frac{t}{6^{3 / 2} \sqrt{\varepsilon}}+3$

Corresponding to the problem with exact solution (15), numerical solution can be seen. Whereas for the case corresponding to the exact solution (16) gives two dimensional plot in Fig. taking $\varepsilon=0.05, x_{1}=4, x_{2}=15$ at times $\mathrm{t}=20,40$. In the next example we have the boundary conditions

$$
u(0, t)=u(4,0)=0, t>0
$$


and the initial conditions shall be derived from the exact solution

$$
u(x, t)=12 \mu(\log E)_{x x}, 0 \leq x \leq 4
$$

Where $\quad E=1+\exp \left(\eta_{1}\right)+\exp \left(\eta_{2}\right)+\left(\frac{\alpha_{1}-\alpha_{2}}{\alpha_{1}+\alpha_{2}}\right)^{2} \exp \left(\eta_{1}+\right.$ $\left.\eta_{2}\right)$

$$
\begin{gathered}
\eta_{1}=\alpha_{1} x-\alpha_{1}^{3} \mu t+b_{1}, \\
\eta_{2}=\alpha_{2} x-\alpha_{2}^{3} \mu t+b_{2}, \\
b_{1}=-0.48 \alpha_{1}, b_{2}=-1.07 \alpha_{2} \\
\alpha_{1}=\sqrt{\frac{0.3}{\mu}}, \alpha_{2}=\sqrt{\frac{0.1}{\mu}} .
\end{gathered}
$$

Further we have the KDV equation of the form

$$
u_{t}+6 u u_{x}+u_{x x x}=0
$$

whose exact solution is given by

$$
u(x, t)=\frac{3+4 \cosh (2 x-8 t)+\cosh (4 x-64 t)}{[3 \cosh (x-28 t)+\cosh (3 x-36 t)]^{2}}
$$

For the last case (17) our solution is plotted in Fig. Taking into account the next case with exact solution given by (20) is shown in Fig. Surface plots showing the solution pro files corresponding to the solutions (15-20)

\section{RESULT AND DISCUSSION}

In this section rigorous study has been made for the solution of KDV equation in the form of wave equation and soliton, in sciences a soliton is a self - reinforcing solitary wave or in other way we can say the wav packets or it is also known as the pulse which never changes it shapes while traveling in the medium ( At constant speed). The soliton came in to act when the motion of in undergo into the dispersive effects or the cancellation of nonlinear effect.

Here the objective of the presented work is not only to understand the soliton solution of the moving pulses and wave but also to introduces the B-spline FEM technique in the above nonlinear equation which is a called as the KDV equation. From the above section wee clearly shows the excellent agreement of the solution obtained w.r.t the B-spline FEM, and now our motivation is give the behavior of the solution for this we have plotted the Fig 1 to 6. In which Fig 1 to 4 ,we shown the interaction of wave solution in one dimensional visualization w.r.t the time space and in the next two fig we plotted the surface(3-D) Fig of the above visualization. From the Fig 1, which is plotted for the different time 4 and 6 ?

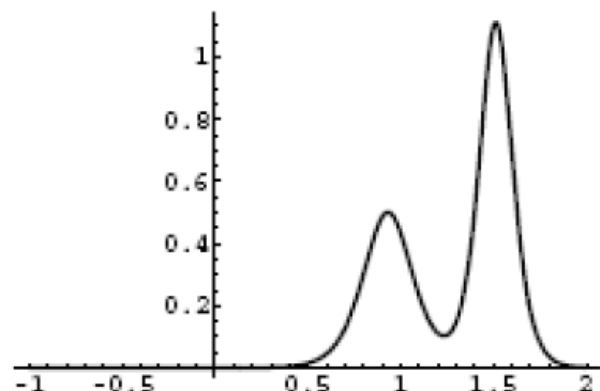

Figure 1A Result obtained at $\mathrm{t}=4$

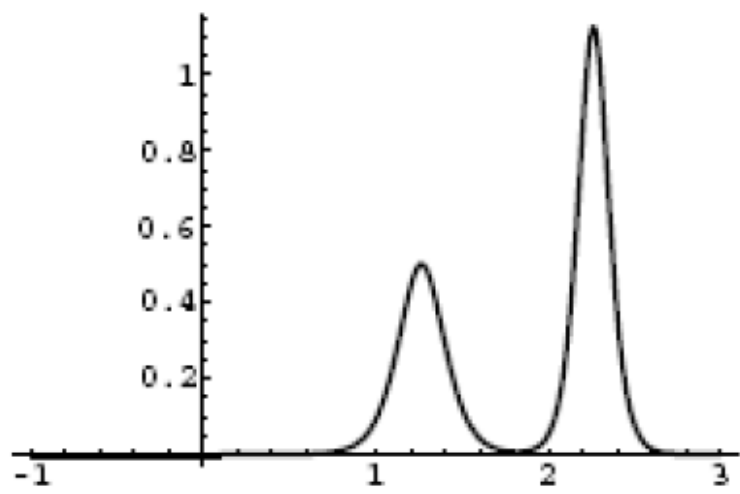

Figure 1B Results obtained at $t=6$

Where we observed that for the small time level that is the wave having a double soliton in the domain $0.5<\mathrm{t}<2$, for the time steps size is maximum 4 , here the another observation we found that the traveling having the maximum magnitude of the velocity is around 1.3in both the cases, but here as we increased the time step the physical phenomena is remain same but it shifted from one point to another which can be seen from the plotted profile.

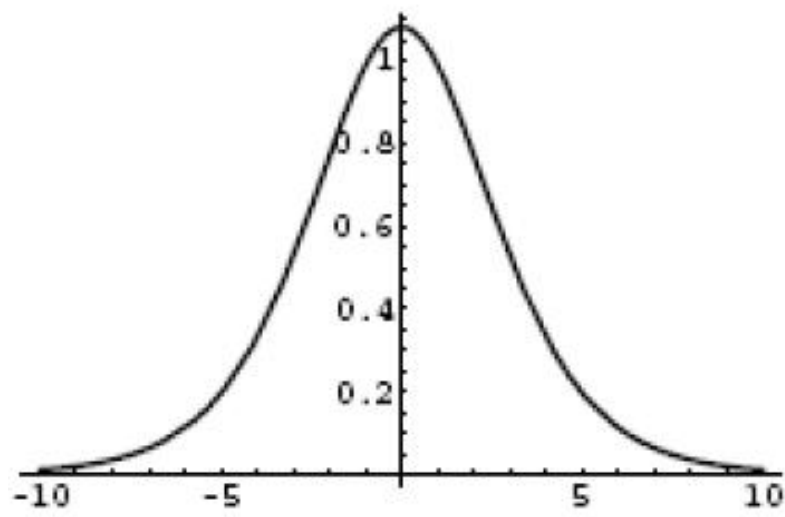

Figure 2 A Results obtained at $t=0$ 


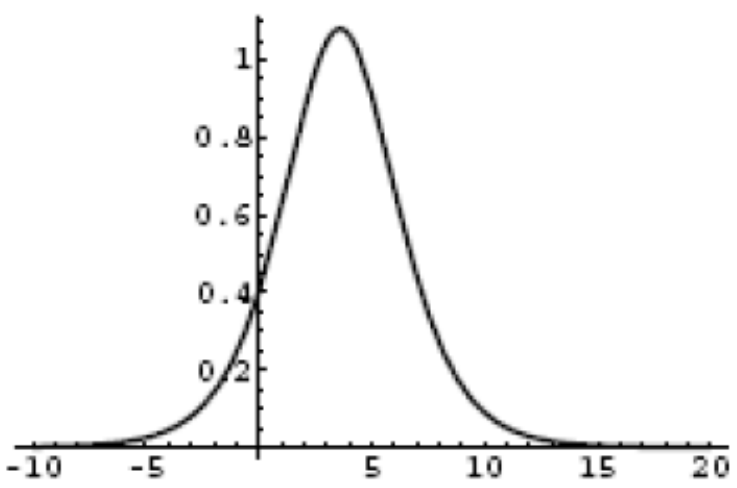

Figure 2 B Results obtained at $t=10$

Now the pattern profile became significantly changes a we increased the time step, basically for the large time it shown the profile of single soliton, here we can visualize from the fig 2 , here the velocity profile has point of maxima $t=0$ and the value is around 1.25 , the maximum value of the velocity has less change but profile having the significant change this might be the cause of the waves pattern because as we increased the time level we get the velocity became less magnitude of the on the other hand we can say that as we increases the time the forcing factor reduces in a time step. This is cause of changes the pattern of the pulses.

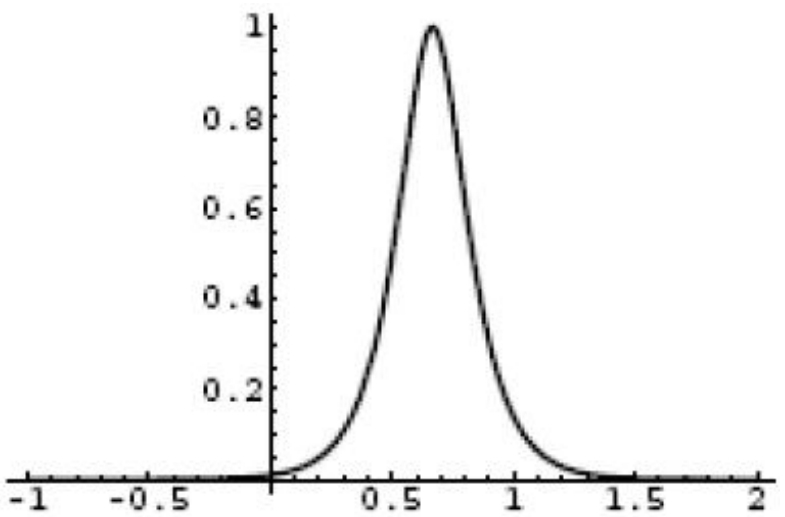

Figure 3 A Results obtained at $t=2$

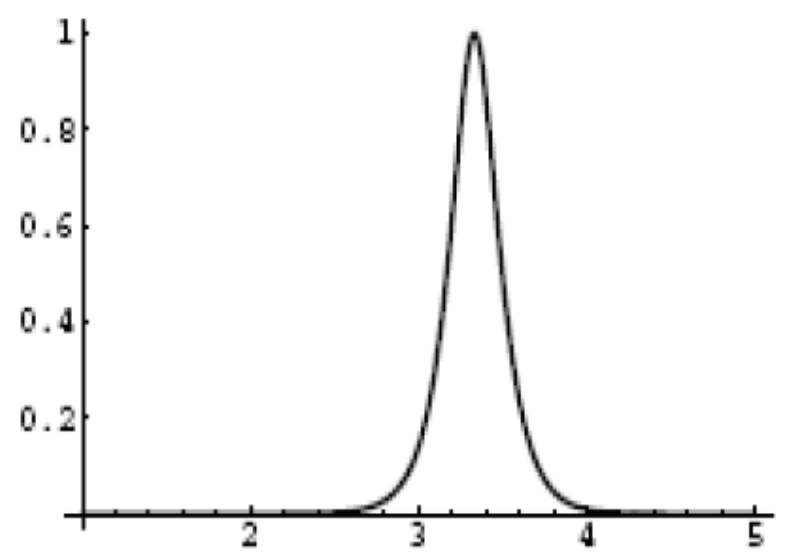

Figure 3 B Results obtained at $t=10$
In the next observation we again reduces the time step for the wave pattern but it is still having the same profile as the previous case but it having the less magnitude of the velocity along with that its peak is shifted from one point to another point, in the time step is again an important observation because in this period waves having the less magnitude along with that, the soliton having a less amount of efficient energy.

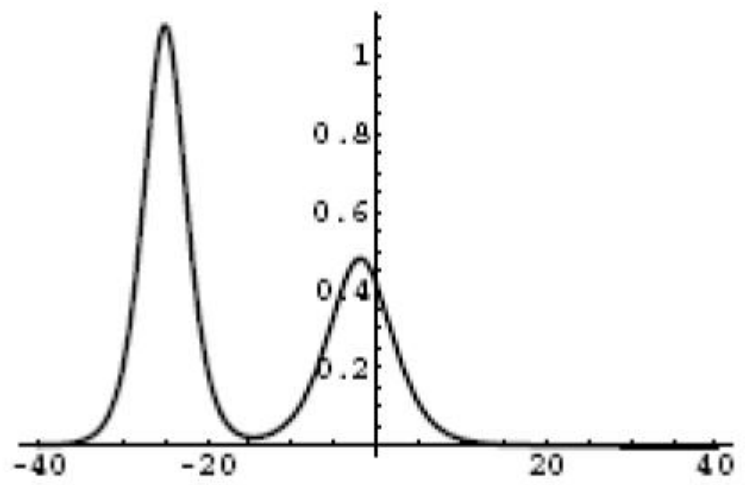

Figure 4A Results obtained at $t=0$

In the fig 4 , we taken large time for the calculation of the wave profile, the successfully we get the unexpected result, that is the profile having the doubly soliton along with that the waves are generated in the reveres time of interval, it is significant result we obtained in this manner, so far the magnitude of the velocity is also changes but it is still close to one.

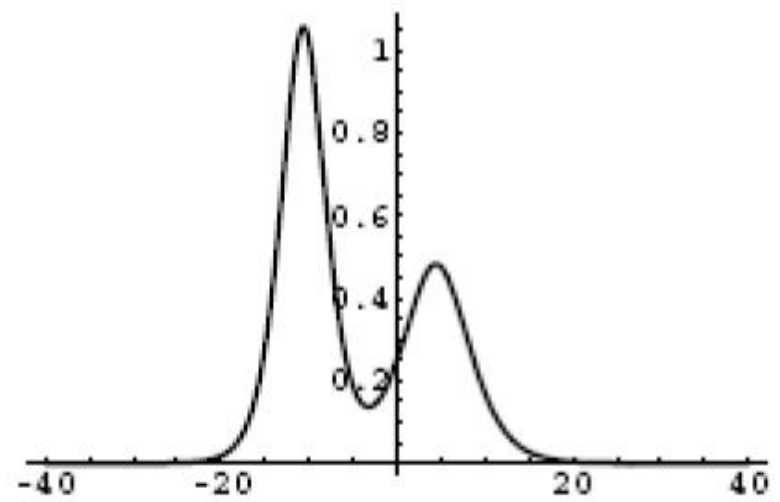

Figure 4B Results obtained at $\mathrm{t}=\mathbf{4 0}$

It means that shock waves hit the target in the same magnitude of the pulses but it changes there profiles in the time interval, it can be clarity seen from the above figs, in order to understand the surface behavior of the physical phenomena so we have attempted the plotting of the 3-D fig of the above phenomena these can be seen in the 5 and 6 

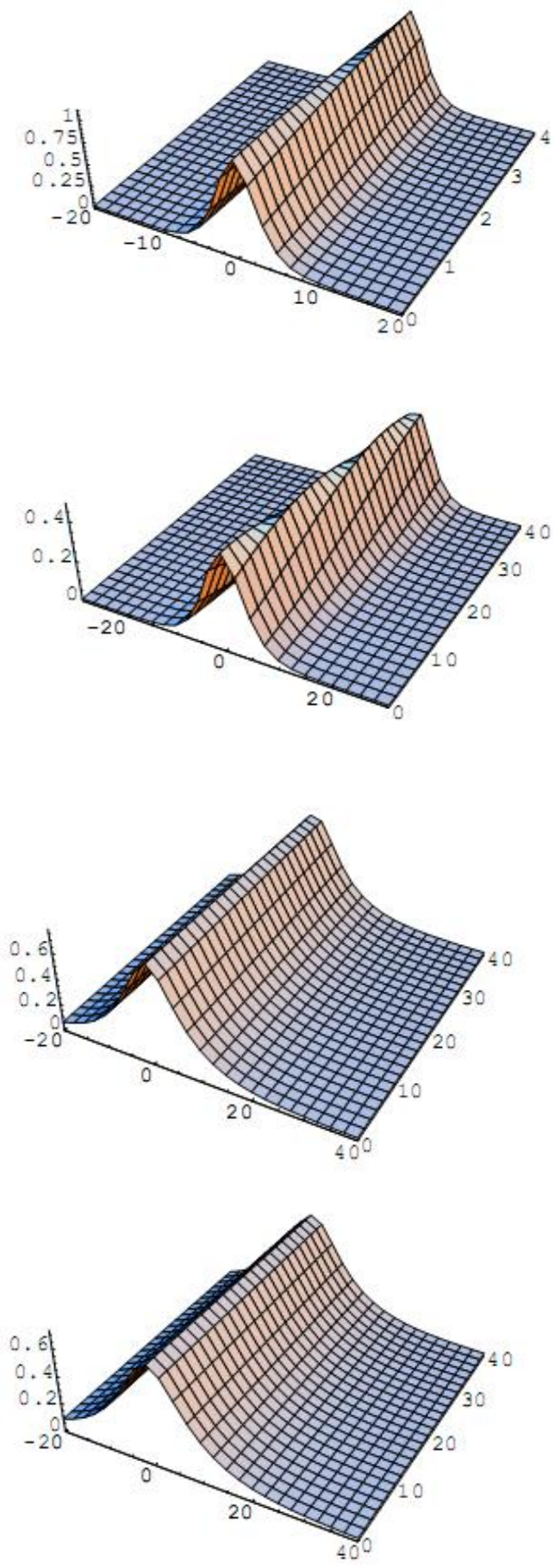

Figure 5A and 5B Numerical solution profile for $p=6$; $c=$ $0: 003 ; \kappa=0.2, p=4 ; c=0: 005 ; \kappa=0.3$

It is an interesting to see the wave profile in the 3-D plot, from the fig 5 (a) and (b), for the fix value of constant wee plotted the singly soliton wave profile. The observation is made with respect to the rigorous time step study here the upper $\mathrm{Z}$ direction is showing the maximum velocity of the traveling waves. The above picture is clearly shows that the maximum magnitude of the velocity is near to one in one case in the other case it having less then which is not found or clearly visible in the two dimensional plot. The changes of the time steps i.e increases the time level is the cause of the decreases the magnitude of the velocity this due to the reduction of the forcing factor in the mean while as we increases the time steps the eternal forces on the waves reduce there power or energy so that we can say that in time space the shock wave through their natural power of hitting the objects. These waves normally occurs near the sea, so it affected the normal life of the human being if it come in less time space, but as time changes it less harmful for the human being . There is some another observation we made in the next fig . As we mention earlier that "solitary-wave pluses" propagating in nonlinear dispersive media so that three behavior is also shows the non linearity in there profile, it can be seen from the fig 6 , here in the last section of our study we analyzed the behavior of the wave propagation for the large time of interval, the study of the above wave propagation basically through the KDV equation so the magnitude of the velocity having no changes.
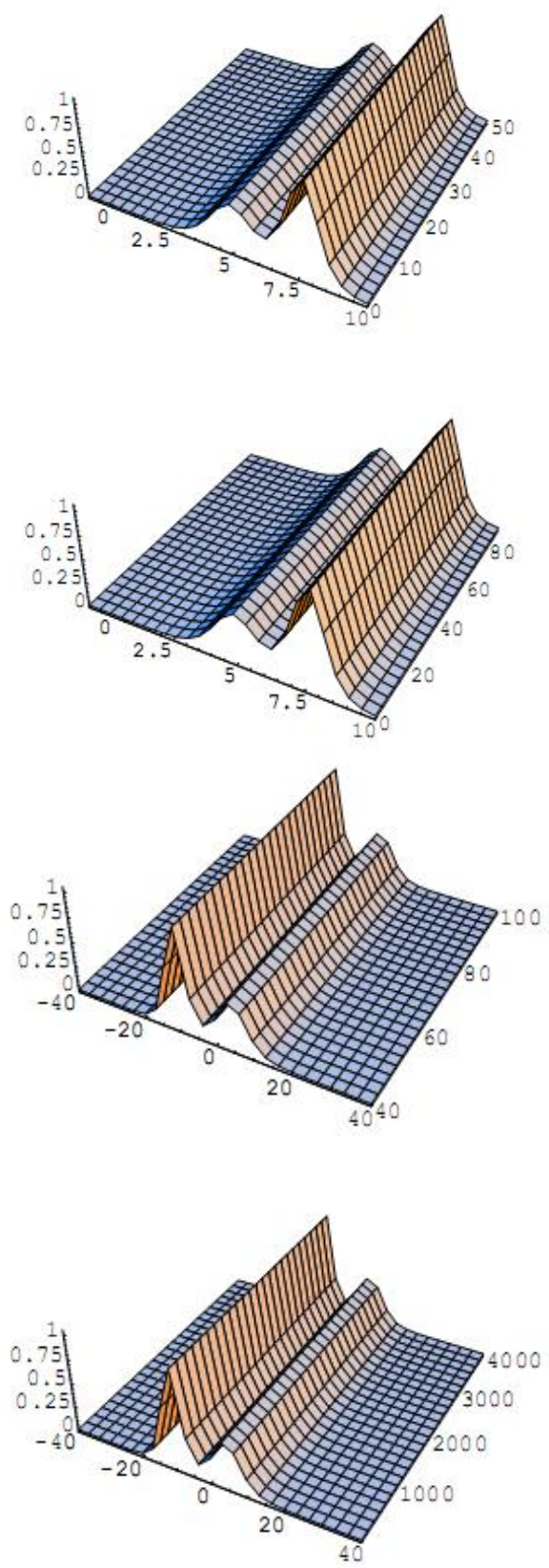

Figure 6A ad 6B Solution profile obtained for interaction of two soliton at $t=100 ; 4000, t=50 ; 90$ 
But the profiles having the drastic changes which we observed from fig 1 to 4 also, but here we can visualize the effect of nonlinearity of the solution, mathematically we can say that the governing PDE is basically non linear in nature so it solution should have a non linear profile this phenomena is being true from the last two fig . The plotted profiles have shown the non linear behavior of the moving pulses and the travelling waves. The important observation is we get that the doubly soliton is occurs for the different time level or steps , which is also great achievement of our study

\section{CONCLUSION}

The present study is basically based on the numerical investigation through the B-Spline FEM for the solution of KDV equation which comes through the waves dynamics the following are conclude of our syudy

1. We get the numerical solution of the non linear problem through FEM

2. B-spline basis function are successfully implemented in the above non linear problem

3. Excellent comparative result we get between the numerical and analytical solution

4. The non linear behavior of the travelling shows the maximum magnitude of the velocity in the above study is around 1

5. In the time space the profile having the significant changes

\section{REFERENCES}

[1] Korteweg D. J., de Vries G., On the change of form of long waves advancing in a rectangular canal, and on a new type of long stationary waves. Phil. Mag. J. Science, 39 (1895) 422-443.

[2] Boussinesq, J. (1877), Essai sur la theorie des eaux courantes, Memoires presentes par divers savants l'Acad. des Sci. Inst. Nat. France, XXIII, pp. 1-680

[3] N.J. Zabusky and M.D. Kruskal, Interaction of solitons in a collisionless plasma and the recurrence of initial states, Phys. Rev. Lett. 6 (1965) 240-243.

[4] Miura, Robert M.; Gardner, Clifford S.; Kruskal, Martin D. (1968), "Korteweg-de Vries equation and generalizations. II. Existence of conservation laws and constants of motion", J. Mathematical Phys. 9: 12041209

[5] Lax, P. (1968), "Integrals of nonlinear equations of evolution and solitary waves", Comm. Pure Applied Math. 21: 467-490,

[6] A. C. Vliegenthart, On finite difference method for Korteweg-de Vries equation,J. Eng. Math., 5 (1971) 137 155.

[7] Miles., John W. (1981). "The Korteweg-De Vries equation: A historical essay". Journal of Fluid Mechanics 106: 131-147.

[8] Dingemans, M.W. (1997), Water wave propagation over uneven bottoms, Advanced Series on Ocean Engineering, 13, World Scientific, Singapore,, 2 Parts, 967 pages

[9] de Jager, E.M. (2006). "On the origin of the Kortewegde Vries equation". arXiv;math/0602661vl [math.HO].

[10] Darvishi, M. T.; Kheybari, S.; Khani, F. (2007), A Numerical Solution of the Lax's 7th-order KdV Equation by Pseudospectral Method and Darvishi's Preconditioning, 2, pp. 1097-1106
[11] A. C. Scott, Y. F. Chu and D. W. McLaughlin, The Soliton: A New Concept In Applied Science, IEEE Proc., 61 (1973) 1443.

[12] Peregrine, D.H., Calculations of Development of an undular Bore, J.Fluid Mech, 25 (1966)321-330.

[13] Malik Zawwar Hussain., Muhammad Sarfraz., Ayesha Shakeel, Shape Preserving Surfaces for the Visualization of Positive and Convex Data using Rational Bi-quadratic Splines., International Journal of Computer Applications ., 27(10), 2011

[14] A. C. Vliegenthart.,On Finite difference method for Korteweg-de Vries equation, J. Eng. Math., 5 (1971)137155 .

[15] Zabusky, N. J.; Kruskal, M. D. (1965), "Interaction of "Soliton" in a collisionless Plasma and the Recurrence of Initial States", Phys. Rev. Lett. 15: 240-243,

[16] K. Abe and 0. Inoue, Fourier expansion solution of the Korteweg-de Vries equation, J. Comp. Phys. 34 (1980) 202-210.

[17] T.R. Taha and M.J. Ablowitz, Analytical and numerical aspects of certain nonlinear evolution equations I. Analytic, J. Comp. Phys. 55 (1984) 192-202.

[18] T.R. Taha and M.J. Ablowitz, Analytical and numerical aspects of certain nonlinear evolution equations III Numerical, J. Comp. Phys. 55 (1984) 231-253.

[19] B. Fornberg and G.B. Whitham, A numerical and theoretical study of certain nonlinear wave phenomena, Phil. Tra. Roy. Sot. London 289 (1978) 373-404.

[20] L. Iskander, New numerical solution of the Korteweg-de Vries equation, Appl. Num. Math. 5 (1989) 215-221.

[21] O.A. Karakashian and W. McKinney, On optimal highorder in time approximations for the Korteweg- de Vries equation, American Math. Sot. 55 (1990) 473-496.

[22] V.A. Dougalis and O.A. Karakashian, On some highorder accurate fully discrete Galerkin methods for the Korteweg-de Vries equation, Math. Comp. 45 (1985) 329-345.

[23] O.A. Karakashian and W. Rust, On the parallel implementation of implicit Runge-Kutta methods, SIAM J. Sci. Statist. Comput. 9 (1988) 1085-1090.

[24] S.Kapoor \& S.Dhawan; " A computational technique for the solution of Burgers' equation" Int. J. of Appl. Math. and Mech. 6(3): 84-95, 2010.

[25] V.Dabral., S.Kapoor., S.Dhawan. S.Rawat “ Finite Element Based solution of Modified Equal Width equation (MEW) with Homogenous Boundary condition using B-spline Basis Function, Chiangmai University International Conference 2011 , Vol. 1, No. 1 (2010) 124 $-131$

[26] V.Dabral., S.Kapoor., S.Dhawan., "Mathematical study of seprated solitry Wave solution for KDV equation: Bspline FEM Approach" . in $4^{\text {th }}$ International conference on "Modeling ,Simulation and Applied Optimization " (ICMSAO-2011) " Kulalumpur (Malaysia), an IEEE conference held at Kula-Lumpur (Malaysia) Paper Published in conference Proceeding Paper code: 96508 in Applied Mathematics Track. P.P 771-775.,

[27] Yogesh Gupta., Manoj Kumar., "A Computer based Numerical Method for Singular Boundary Value Problems., International Journal of Computer Applications, 30(1)., 2011, pp. 21-25 